\title{
METHODS OF INTERPRETATION OF DATA FROM ISOKINETIC TESTS AND MRI STUDIES DURING REHABILITATION OF PATIENTS AFTER RECONSTRUCTIVE SHOULDER JOINT SURGERY
}

\author{
E.M. Kabaev ${ }^{1, *}$, Yu.A. Hamad ${ }^{2}$, K.V. Simonov ${ }^{3}$, A.G. Zotin ${ }^{4}$ \\ ${ }^{1}$ Federal State-Financed Institution Federal Siberian Research Clinical Centre, the Federal Medical Biological Agency, \\ 26 Kolomenskaya st., 660037, Krasnoyarsk, Russian Federation - kabaevem@mail.ru \\ ${ }^{2}$ Institute of Space and Information Technologies, Siberian Federal University, 79 Svobodny st., Krasnoyarsk 660041, Russian \\ Federation - y.albayati8@gmail.com \\ ${ }^{3}$ Institute of Computational Modelling of the SB RAS, 50/44 Akademgorodok, Krasnoyarsk 660036, Russian Federation - \\ simonovkv@icm.krasn.ru \\ ${ }^{4}$ Institute of Computer Science and Telecommunications, Reshetnev Siberian State University of Science and Technology, \\ 31 Krasnoyarsky Rabochy ave., Krasnoyarsk, 660037, Russian Federation - zotin@ @ibsau.ru
}

\author{
Commission V, WG II/10
}

KEY WORDS: Shoulder joint, Rehabilitation, Robotic mechanotherapy, CON-TREX, Biomechanics, Numerical data modeling, Visualization, Correlation analysis.

\begin{abstract}
:
The research results in the field of computer visualization of the shoulder joint biomechanics are presented. The possibilities of using biomechanical robotic mechanotherapy on the CON-TREX complex in the rehabilitation treatment of patients after arthroscopic shoulder surgery are shown. The possibilities of additional visualization of magnetic resonance imaging (MRI) data using spectral decomposition methods (Shearlet transform and contrasting with color coding) are studied. Experiments with the use of the proposed diagnostic technique are described. The relationship between the MRI data and CON-TREX protocols in planning and implementation of the rehabilitation procedures is demonstrated. The technique which allows to improve the quality and availability of the MRI data in the study of the shoulder joint biomechanics during restorative treatment is described.
\end{abstract}

\section{INTRODUCTION}

Orthopedic biomechanics was designed to study and target the optimization of mechanical stresses that affect normal bones, joints and soft tissue that have been altered by disease, injury, or have been surgically treated. The strategy for leading experimental research in this field includes a combination of orthopedic surgery, mechanical testing and medical imaging (Zdero, 2017). The creation of optimal physical models of the shoulder joint with imitation of muscles and other components in real scale is a relevant topic in modern science (AlmeidaGallaraga et al., 2017). Back in 2002, a model of the shoulder joint was developed to analyze the effect of the shape of the humeral head on the distribution of stress in the scapula during various physiological movements (Buchler et al., 2002).

Modern rehabilitation of patients who underwent surgical interventions on joints is associated with advances in the study of their biomechanics and modern diagnostic MRI methods. Comprehensive assessment methods allow restorative medicine specialists to obtain the most complete understanding of the dynamic and static components of the shoulder joint work. The correct objective assessment of the functional capabilities of the shoulder joint is also important both before the start of rehabilitation and in the process of it.

The shoulder forms the basis for movement of the entire upper limb. In the context of modern surgical capabilities, including arthroplasty, mathematical modeling is of particular value at all stages from planning the operation to the completion of postoperative rehabilitation. The complex process of ensuring mobility and dynamic stability is studied in a multidisciplinary manner. And yet, the methodology for describing and forming mathematical models in statics and dynamics already exists today, being the work of a huge number of scientists (Nedoma et al., 2011).

Dynamic kinematics of the shoulder joint is studied on the basis of radiographs and CT data with the aim of spatial modeling of normal and pathological biomechanics of movements, facilitating the diagnosis of pathology and planning of upcoming reconstructive surgical interventions (Kozono et al., 2018.

Such studies contribute to the elimination of the routine approach to data interpretation by additional methods, such as computed tomography. Thus, a relationship is being established

\footnotetext{
* Corresponding author
} 
between radiologists and surgeons, who expect better baseline data on the patient's morphological characteristics and the type of biomechanical disorder before planning the reconstruction of damaged structures (Yun et al., 2018).

Measuring dynamic shoulder kinematics in vivo is critical to improving understanding of multiple pathologies. Various motion capture systems using markers on the skin show the movements of segments, bones (osteokinematics), but do not imply detailed visualization of arthrokinematics inside the joint. However, their combined use with additional visualization of MRI data created the prerequisites for a better analysis within the framework of non-invasive methods for studying kinematics in motion.

Certain modern studies are aimed at the development of special complex accurate methods of analysis. The creation of reference kinematic models of the joint, primarily three-dimensional, is the result of their work (Charbonnier et al., 2014) and (Massimini et al., 2011). This is especially important when the injury or disease is old or chronic and results in persistent pathobiomechanical changes.

In such cases a decision is made on the technique of the operation, its timing and tactics of physical rehabilitation before and after reconstructive intervention. This is especially true in relation to total shoulder arthroplasty after a fracture. It is required to identify and standardize specific biomechanical parameters (Mitkovski, 2020). Using morphometric techniques, data were obtained on the anatomical features of the shoulder joint in various racial groups, which are also useful for clinicians to consider (Cabezas et al., 2016).

Thanks to the accumulation of data on mathematical modeling of biomechanics and video analysis of movements, the research has moved to the next level, allowing the description of the coordinated consensual movements of the scapula, clavicle and humerus, as well as the capsular-ligamentous and tendon components. It became possible to study motor rhythms, for example, in describing the movement of abduction in the shoulder-blade joint. Disorders of the shoulder-scapular rhythm are observed in the vast majority of pathologies and injuries of the shoulder joint. Research data indicates the importance of the robotic component in the study of biomechanics.

No one doubts the effectiveness of robotic technologies in rehabilitation. However, they were developed on the basis of simple biomechanical models of movement, therefore, they require additions for better rehabilitation and improvement of its strategies (Zhibin et al., 2018). The improvement in the quality of all diagnostic methods used in orthopedics and traumatology is gaining momentum every year. The main goal of researchers is to eliminate the routine approach to diagnostics and fill the work of specialists with multidisciplinary knowledge. Indeed, even such standard methods as joint ultrasound do not lose their relevance (Precerutti et al., 2020).

The accents of pression of the articular surfaces are investigated on special models, like Anybody, which is an attempt to describe arthrokinematics in the joint non-invasively. Models of arthropathies using the example of rotator cuff injuries make a great contribution to understanding the essence of pathological changes in the musculoskeletal system. Improvement requires the accuracy of these models and their integration into such techniques as CT and MRI. All of the above developments can be used to create expert systems (Lemieux et al., 2012).

\section{RELATED WORK}

The active development of arthroscopy in reconstructive surgery of the shoulder joint has been observed for the last 20 years. The benefits of arthroscopic reconstruction of joint structures include small skin incisions, improved visualization and assessment of joint and rotator cuff defects, improved mobilization and release of the rotator cuff, reduction in postoperative pain and joint stiffness, and improved rehabilitation potential. The disadvantages include the technical complexity of execution and not always consistent tendon-bone fixation. The studies of Wolf et al. show that $94 \%$ of patients with rotator cuff reconstruction receive good and excellent recovery results (Cioppa-Mosca et al., 2008). Rehabilitation requires a long healing time after surgical tissue repair with adequate loads in rehabilitation. Typically 5 phases of rehabilitation treatment take up to 24 weeks (Cioppa-Mosca et al., 2008).

Biomechanical analysis helps to maintain and improve the initial rehabilitation potential in rehabilitation through therapeutic and diagnostic techniques such as robotic mechanotherapy with biofeedback and a variety of systems for capturing and analyzing movements in 3D (Hamill et al., 2015).

In addition to the static and dynamic components used to ensure stable joint operation it is important to take into account the rhythms of movements in the joints of the upper shoulder girdle. The correct shoulder-scapular rhythm, coordinated and consensual movement of the scapula, acromioclavicular, sternoclavicular and scapular-shoulder joints ensure normal movements of the upper shoulder girdle which should be the object of studies in biomechanics and rehabilitation (Brukner and Khan, 2012).

Considering the above there is a need to develop and introduce into practice a methodology that allows for a comprehensive assessment of the state of all components that characterize the quality and functionality of joint structures biomechanics at any given time. The study of biomechanics focuses on the study of 2 basic components: osteokinematics (the movements of the bones that form the joint) and arthrokinematics (the interaction of their articular surfaces in the joint itself) during movement. They are considered interrelated in the study of norm and pathology (Gorniak, 2016).

\section{METHODS}

Biomedical imaging is very important for life sciences and health care. Many innovations in biomedical imaging are fundamentally related to the mathematical sciences. Mathematical analysis ensures that the constructed algorithm serves its purpose. Software based on these techniques supports effective guidance for imaging procedures such as biopsy, noninvasive surgery planning, and radiation therapy. Mathematical models (methods) are basic for the construction of all visualization methods. Quantitative image reconstruction lies in the basis of such methods as CT and MRI (Pal Kaur S., 2016).

Thanks to the additional visualization, the doctor can noninvasively monitor the healing process of damaged or pathologically altered body tissues. In medical imaging, these different imaging techniques are called modalities. Anatomical 
modality provides insight into anatomical morphology. These include x-rays, ultrasonography or ultrasound, computed tomography, and magnetic resonance imaging. Computational algorithms that complement visualization form modality. Mathematical methods and algorithms directly affect some of the main problems in this area, including image enhancement, registration and segmentation (Angenent et al., 2006).

Most real-world images are inherently low-dimensional in nature. This property of sparsity can be detected by presentation systems from the field of applied harmonic analysis, such as wavelets or shifts. The task of improving imaging, such as MRI, is then solved using sparse regularization, which in certain situations is called compressed probing (Kutyniok et al., 2018). Experiments are known on the use of dynamic MRI for improved visualization and understanding of arthrokinematics of the shoulder joint in rotator cuff injuries (Tempelaere et al., 2016).

The study of the geometry of the joint is carried out, including using the MRI data, with additional morphometric techniques. From the study of the position of the humeral head relative to the center of rotation, they are constantly supplemented with new indicators describing arthrokinematics, without a separate emphasis on soft tissues (Kelkar et al., 2001) and (Manoj et al., 2020).

The paper reflects information about conducted assessments of the main parameters of the protocols of isokinetic testing of the shoulder joint (power, torque, work, range of motion). The protocol is formed by the system of the biomechanical training complex CON-TREX. Additionally, MRI images as well as clinical and functional indicators of goniometry (measurement of the angle of motion in degrees) were studied during active flexion-extension abduction-adduction and rotation in the shoulder joint. Clinical and functional data were analyzed by statistical methods to compare the dynamics at different stages of rehabilitation. Taking the torque parameter from the dynamics reports as a basis, a three-stage study was carried out using mathematical methods.

After the preliminary processing and formation of motion models the visualization was performed using the method of multivariate histograms and multivariate correlation analysis (Zotin et al., 2020). Correlation between the performance criteria and various characteristics of a parameter and crosscorrelations between parameters are set in a correlation matrix. The correlation coefficients are then used to identify which parameters have a net effect on the performance of the test (Barlett R., 2005).

Mathematical modeling is supplemented by the histogram method, which reflects the energy component of the studied movements. The dynamic motion profile graphically displays all the studied parameters in aggregate and superimposed on each other when they are repeated many times.

Additionally, MRI images as well as the clinical and functional indicators of goniometry (measurement of the angle of motion in degrees) were studied during active flexion-extension abduction-adduction and rotation in the shoulder joint. The series of MRI images were studied using Shearlet transform and color-coding algorithms.

In our proposed methodology, an important place is given to the study of the dynamic profiles of movements observed in the system of the CON-TREX biofeedback simulator. The assessments of the main parameters of the protocols of isokinetic testing of the shoulder joint (power, torque, work, range of motion) were conducted.

The homogeneity and density in the tissue structure after the reconstruction is satisfactory with small areas of cicatricial reconstruction. Stabilization of the glenoid lip after the surgical treatment, a more correct ratio between the articular surfaces of the scapula and humerus with the correct placement of implants (screws) in the articular process, characterizes a favorable course of the postoperative remodeling of the joint structure (Fig. 1) (Malanin et al., 2016; Sims et al., 2008).
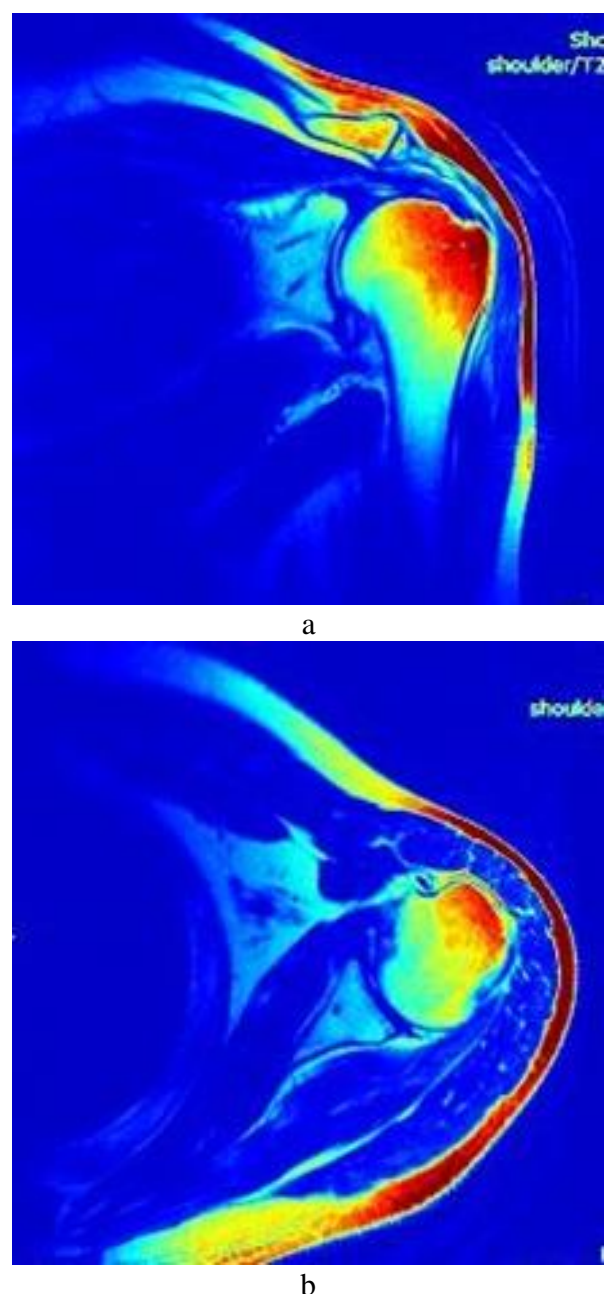

Figure 1. An example of visualization of MRI images after surgery. $a-$ frontal view, $b-$ axial view of the MRI of the shoulder joint after Latarget plasty

The function of the shoulder is the result of a complex interaction between the work of the bone and soft tissue components, passive and active stabilizers. When moving in the shoulder-shoulder joint, the head of the humerus should exert pressure on the glenoid of the scapula with a relatively constant force and, due to the coordinated work of the muscles, maintain a central position throughout the entire range of motion and at any effort. This is important to take into account when assessing $\mathrm{X}$-ray anatomy, biomechanics and a comprehensive study of the interaction between these stabilizing systems (Dickens et al., 2021). 
As the main method of rehabilitation for the study group, we used robotic mechanotherapy on the CON-TREX training complex with biofeedback, since it is equipped with an isokinetic dynamometer. The very reconstructive technique during arthroscopic operations is aimed at restoring the structure of the predominantly static component of joint stabilization (bones, ligaments, tendons, capsules). Muscle work is coordinated by the training process on CON-TREX in isokinetic and CPM modes with any of the possible types of muscle resistance.

When setting up the system, before starting work, the limbs are provided with a safe physiological position on a special adapter and it is possible to simultaneously perform not only the linear, but also rotational (spiral) movements in the segment (Fig. 2).

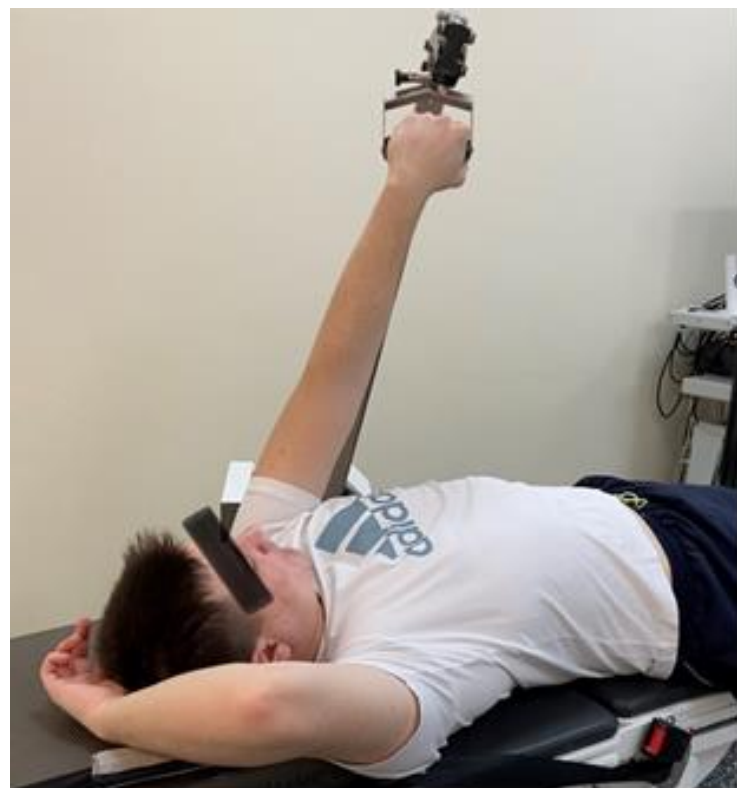

Figure 2. Flexion-extension with simultaneous rotation during robotic mechanotherapy on CON-TREX

It is this that does not violate the static component, stimulates the dynamic one and makes it possible for the patient's nervous system to realize the safety of the movement being made. Such a therapeutic technique effectively eliminates one of the most formidable complications of injuries and diseases of the joints kinesiophobia (fear of movement), since the awareness of the safety of movement occurs when the patient is actively involved in the training process.

CON-TREX system captures the parameters that determine the conditions for the exercise and calculates many statistical indicators that characterize the physical parameters of the exercise by the patient. The software of the medical system visualizes the basic data (Fig. 3). The graph of the Torque $(\mathrm{N} \cdot \mathrm{m})$ versus time $(\mathrm{sec})$ is shown in the upper left area, the graph below shows the Position (deg) versus time (sec), and the figure on the right shows graphs of all 50 cycles of the dependencies of the Torque (N.m) on the Position (deg).
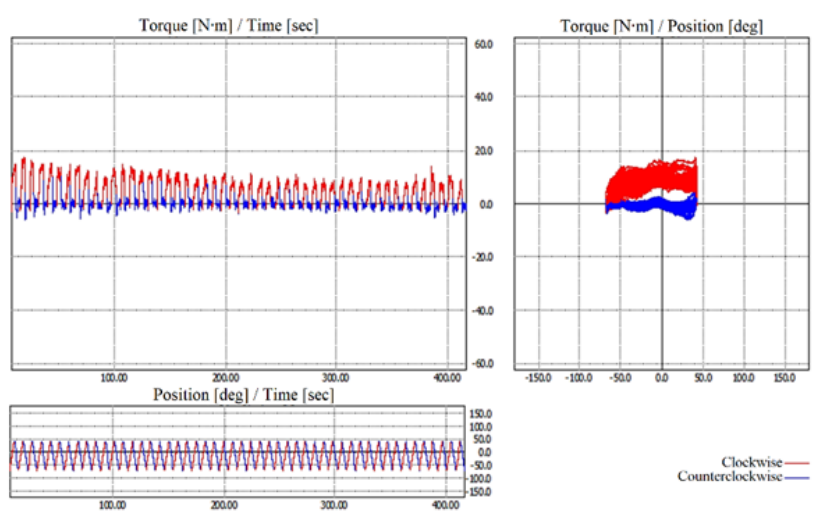

Figure 3. Data visualization plane of the software tool

Because of the feedback, the simulator system is easily adapted to the patient's functional capabilities. A special active compensation mode helps to carry out movements even the most weakened patients. Standard parameters (torque, power, ROM, total work) are formed into dynamics protocols for each perfect approach to the exercise.

The specified main parameter in isokinetics is the speed of movement, which is very important in relation to the rehabilitation process, because it must correspond to the phase of the treatment process and the permissible loads corresponding to the time after surgery (Payton et al., 2008).

\section{EXPERIMENTS}

The experiments were carried out on the basis of the FSRCC FMBA of Russia on the control group of 50 people who underwent arthroscopic surgery for injuries of the shoulder joint structures. Comparative analysis of the constructed models made it possible to study the features of the complex use of mechanotherapy and its influence on the rate of regression.

Clinical and functional data were processed by means of the statistical methods for a quantitative comparison of the dynamics at different stages of rehabilitation. Based on the study of the torque from the dynamics reports, a three-stage study of the data was carried out using mathematical methods. After the preliminary processing and formation of motion models (clockwise and counterclockwise), visualization was performed using the methods of multivariate histograms and correlation analysis with color visualization of the dynamics of the obtained data (Fig. 4). 


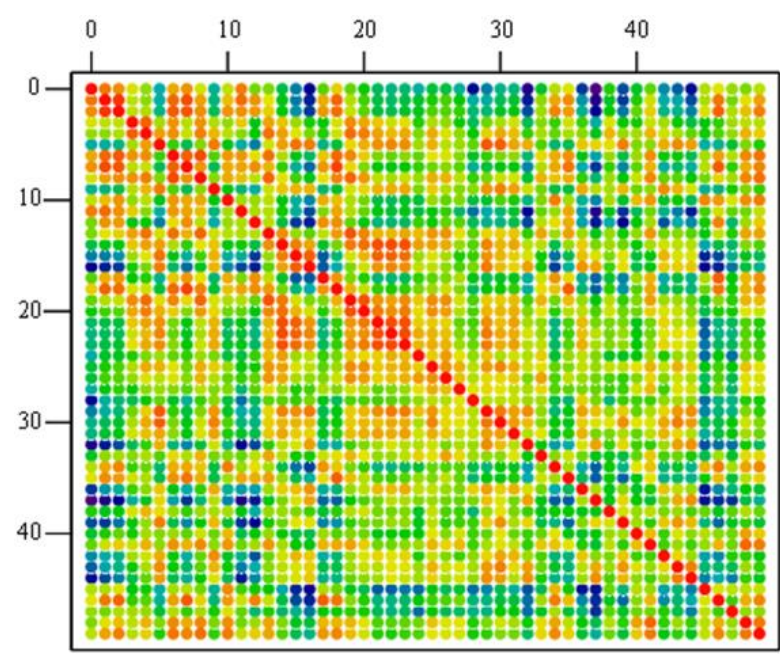

Figure 4. Example of a correlation matrix of 50 repetitions when performing an exercise on CON-TREX

Also, within the framework of the research, a series of MRI images of patients were studied. The above-described image analysis technique was used, including algorithms for Shearlet transformation and contrasting of the selected structures which are objects of interest with color coding.

There is a study in which the selection of the most optimal and safe exercises and the type of resistance for the shoulder joint were selected in accordance with the achieved range of motion (Haberle et al., 2018). On the example of a patient in the study group, we obtained the following results: relief of pain in the first month of rehabilitation, satisfactory muscle strength and joint mobility in full with the ability to perform complex coordination and balance exercises (Fig. 5). Restoration of reflexes during manual muscle testing was observed by the end of the 3rd month from the moment of surgical treatment. According to goniometry data, complete recovery of shoulder joint mobility in patients of the study group was noted by the end of the 2 nd month of rehabilitation treatment, provided that active movements were performed.

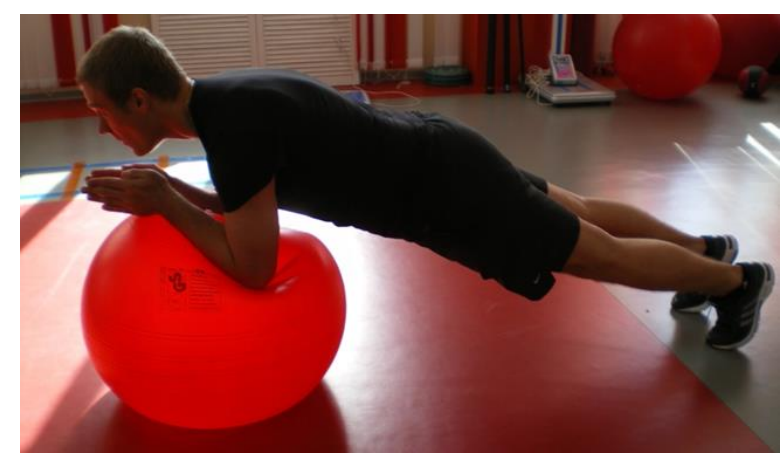

Figure 5. Exercise for coordination on a fitball in a patient after 2 months of rehabilitation
The goniometry method was used to measure the range of motion (ROM - Range of motion) in degrees from 0 to 180 . The control periods were 1 day of the beginning of rehabilitation, then repeated measurements after the 1 st, 2nd and 3rd months. According to goniometry data, complete restoration of shoulder joint mobility in patients of the study group (group I) was observed by the end of the 2nd month of the rehabilitation treatment, provided that active movements were performed (Fig. 6).

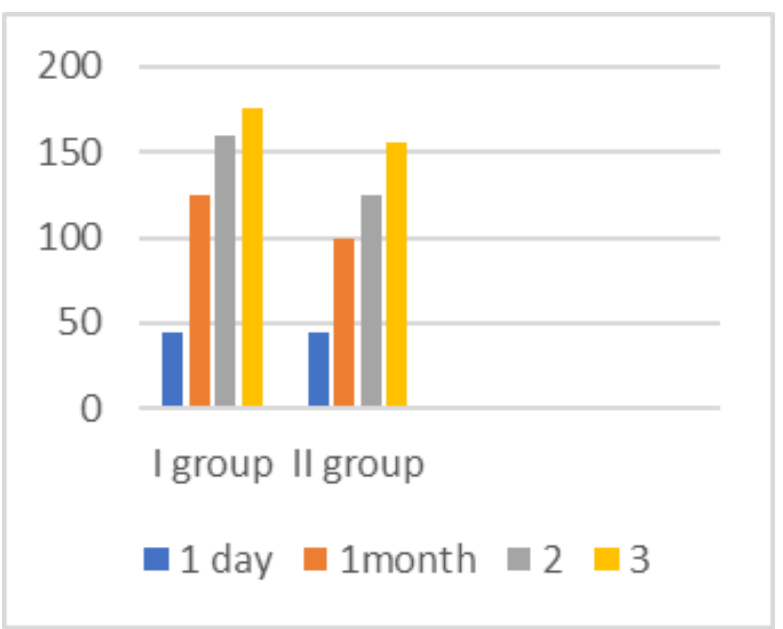

Figure 6. Dynamics of ROM flexion in degrees by months of rehabilitation

In the course of a comparative quantitative analysis, the corresponding approximation models and histograms constructed on the basis of the dynamics data made it possible to evaluate in detail the effectiveness of the effect of mechanotherapy on the functional activity of the shoulder joint (shape, position, and magnitude of the scatter of the histogram). The created set of correlation matrices (color coded) based on indicators from dynamic reports characterizes a specific functional model of the joint at a certain stage of observation.

The dynamics of the severity and frequency of clinical symptoms of the pathological process in different periods was also studied. The constructed to quantify the effectiveness of the effect of mechanotherapy on the functional activity of the shoulder joint. Methods of additional visualization of the MRI images made it possible to increase the accuracy of diagnostic assessments of the state of shoulder joint structures due to selective and detailed display of their density and uniformity (Fig. 7). 
a
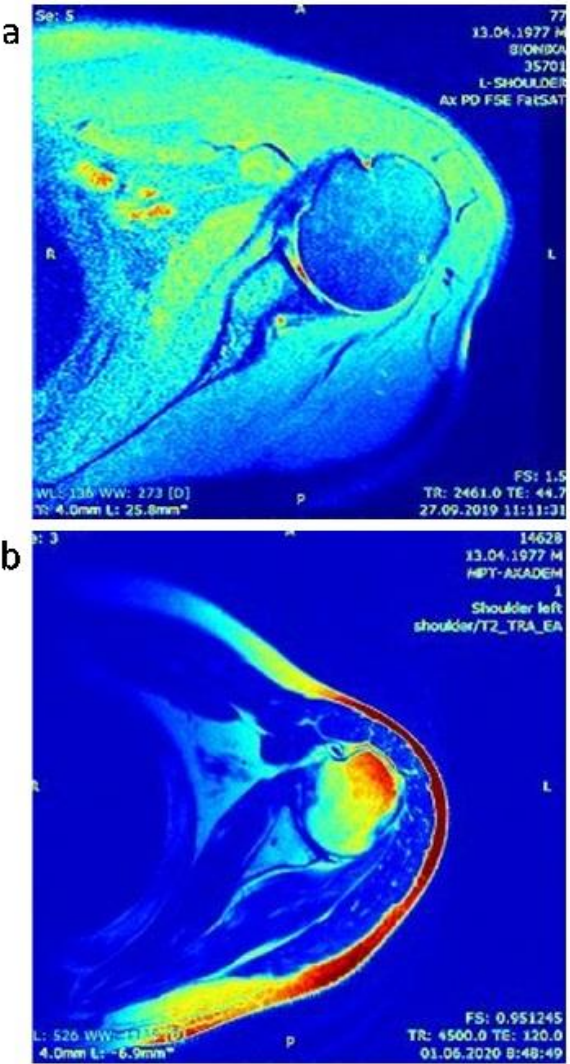

Figure 7. Examples of the MRI image process
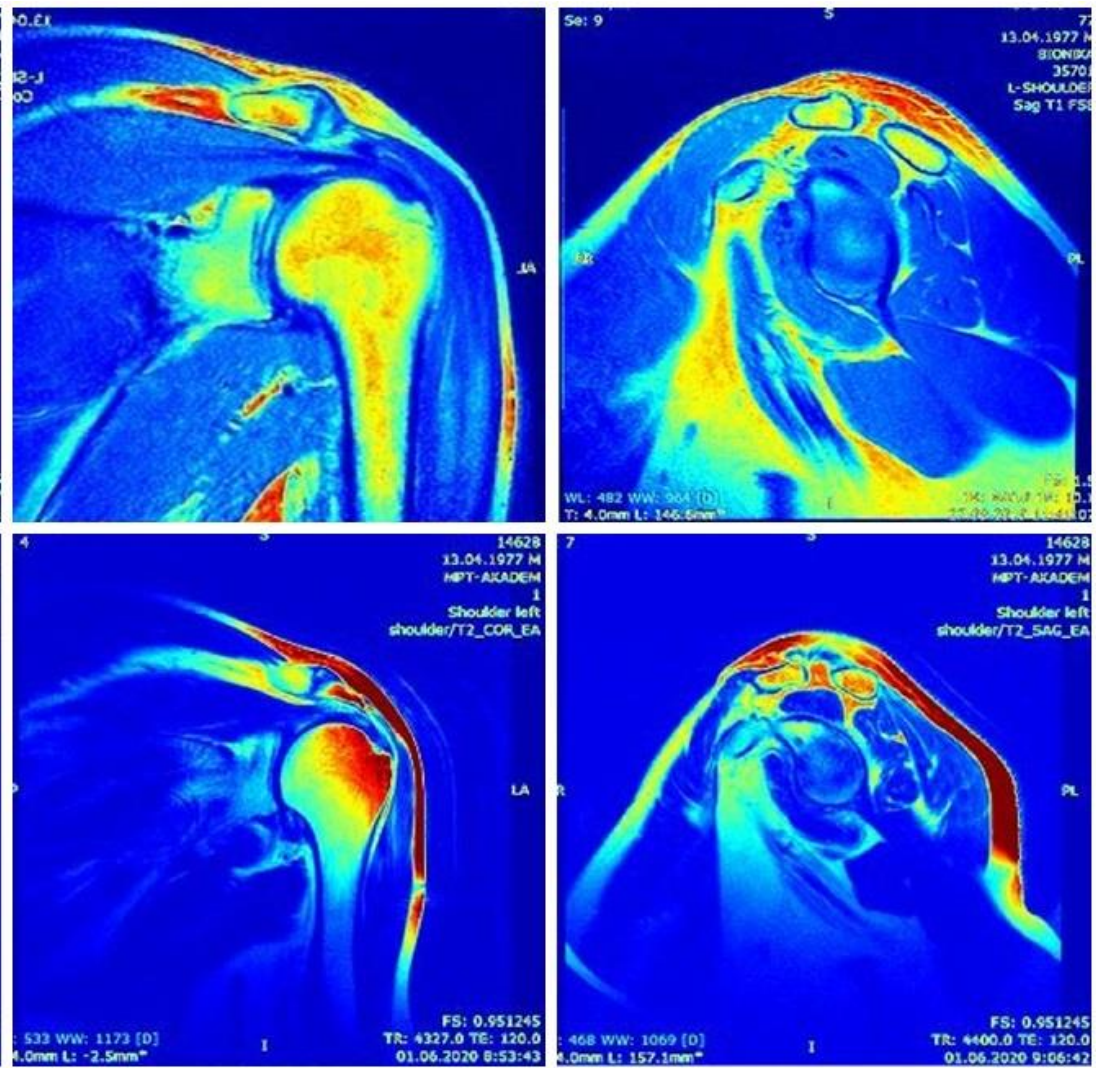

surgery; (b) 1 month after surgery
surith waP injury and partial

\section{DISCUSSION}

Models and indicators in normal and pathological state at different stages of rehabilitation or testing make it possible to assess the direction of functional remodeling of the shoulder joint and the degree of its arthrokinetic stabilization.

Enhancement of the visual representation of the entire set of initial experimental clinical data CON-TREX allows to improve the accuracy of diagnostic assessments by interpreting statistical indicators both in kinematics and in dynamics. A complex technique of biomechanical robotic mechanotherapy with additional data visualization provides the necessary information to specialists in sports medicine and rehabilitation in an accessible form and may well become a component of expert systems in these areas.

Its use is possible in the examination of the functional capabilities of joints including after their endoprosthetics for a more accurate study of the processes of remodeling of bones and periarticular structures. This will allow to identify the instability of the components of the endoprosthesis at an early stage and to assess the consistency of implants during reconstructive operations.

\section{CONCLUSION}

The obtained data reflect the state of all components of the unified kinematic model of the shoulder joint at a particular moment in the rehabilitation process. The proposed complex approach significantly improves the quality of the step-by-step diagnostics for injuries of the shoulder joint which opens up prospects for its application in biomechanics and practical medicine.

Thus, comparing the usefulness of the restoration of dynamic (functional) and static (morphological) equilibrium, we assess the degree of adaptation and the potential of the patient's body to restore the lost functional capabilities. A patient starting rehabilitation, after removing stitches and short-term immobilization, having MRI studies on hand after surgery, can be better examined during the initial testing using Con-trex, with additional data visualization. As a result, the rehabilitation process becomes more predictable and safe, and the planning of each phase is more reasonable.

\section{REFERENCES}

Almeida-Gallaraga, D., Ros-Felip, A., Alvarez-Sanchez, V., Marco-Martinez, F., Serrano-Mateo, L., 2017: Kinematics based physical modelling and experimental analysis of the shoulder joint complex. Ingeneria e Investigacion 37(3), 115123.

Angenent, S., Pichon, E., Tannenbaum, A., 2006: Mathematical methods in medical image processing. Bulletin (New Series) of the American Mathematical Society 43(3), 365-396.

Barlett, R., 2005: Sports Biomechanics. Reducing and improving Performance. Taylor \& Francis e-library. 
Brukner, P., Khan, K., 2012: Brukner \& Khan's Clinical Sports Medicine. 4th.ed. McGraw-Hill Australia Pty Ltd.

Buchler, P., Rakotomanana, L., Farron, A., 2002: Virtual power-based algorithm for decoupling large motions from infinitesimal strains: application to shoulder joint biomechanics. Computer Methods in Biomechanics and Biomedical Engineering 1.5(6), .387-396.

Cabezas, A.F., Krebes, K., Hussey, M.M., Santoni, B.G., Kim, H.S., Frankle, M.A., Oh. J.H., 2016: Morphologic Variability of the Shoulder between the Populations of North American and East Asian. Clinics in Orthopedic Surgery 8, 280-287.

Charbonnier, C., Chague, S., Kolo, F.C., Chow, J.C.K., Ladermann, A., 2014: A Patient-Specific Measurement Technique to Model Shoulder Joint Kinematics. Orthopaedics \& Traumatology Surgery \& Research 100(7), 715-719.

Cioppa-Mosca, J., Cahill, J.B., Cavanaugh, J.T, Corradi-Scalise, D., Wolf, A., 2008: Handbook of Postsurgical Rehabilitation. Guidelines for the Orthopedic Clinician. Mosby.

Dickens, J.F., Owens, B.D., 2021: Shoulder instability in the athlete: management and surgical techniques for optimized return to play. SLACK Incorporated.

Gorniak, G., 2016: Upper and lower Extremity Biomechanics. Biomechanics for health sciences - A study part 1. 1st ed. Gerard Gorniak \& bookboon.com.

Haberle R., Schellenberg F., List R., Pluss M., Taylor W.R., Lorenzetti S., 2018: Comparison of the kinematics and kinetics of shoulder exercises performed with constant and elastic resistance. BMC Sports Science, Medicine and Rehabilitation 10(1), 1-13.

Hamill, J., Knutzen, K.M., Derrick, T.R., 2015: Biomechanical basis of human movement. 4th ed. Wolters Kluwer.

Kelkar, R., Wang, W., Flatow, E., Newton, P., 2001: Glenohumeral mechanics: A study of articular geometry, contact, and kinematics. Journal of Shoulder and Elbow Surgery 10(1), 73-84.

Kozono, N., Okada, T., Takeuchi, N., Hamai, S., Higaki, H, Shimoto, T., Ikebe, S., Gondo, H., Nakanishi1, Y., Senju, T., Nakashima Y., 2018: Dynamic kinematics of the glenohumeral joint in shoulders with rotator cuff tears. Journal of Orthopaedic Surgery and Research 13(1):9.

doi: 10.1186/s13018-017-0709-6.

Kutyniok G., Ma J., März M., 2018: Mathematical Methods in Medical Image Processing. Quantification of Biophysical Parameters in Medical Imaging 153-166.

Lemieux, P., Nuco, N., Hagemeister, N., Tetreault, P., 2012: Mechanical analysis of cuff tear arthropathy during multiplanar elevation with the AnyBody shoulder model. Clinical biomechanics 27(8), 801-806. Bristol, Avon.

Malanin, D.A., Tregubov, A.S., Grunin, S.V., Cherezov, L.L., 2016: The effect of the location and remodeling of the bone graft block on the results of the Bristow - Latarjet operation. Traumatology and Orthopedics of Russia 22 (3), 31-43.
Manoj, M., Kashikar, S.V., Dhande, R.P., ,2020: MRI based morphometry of shoulder joint. International Journal of Psychosocial Rehabilitation 24(8), 4267-4275.

Massimini, D.F., Warner, J.J., Li, G., 2011, Non-invasive determination of coupled motion of the scapula and humerusAn in-vitro validation. J. Biomech, 44(3), 408-12.

Mitkovski , I., 2020: Biomechanical principles of shoulder joint as a basis of post fracture endoprosthesis replacement. Journal of IMAB 26(1), 2910-2915.

Nedoma, J., Stehlik, J., Hlavacek, I., Danek, J., Dostalova, T., Preckova, P., 2011: Mathematical and computational methods in biomechanics of human skeletal systems: an introduction. John Wiley \& Sons, Inc.

Pal, K.S., 2016: Contribution of Mathematical Models in Biomedical Sciences - An Overview International. Journal of Applied Science-Research and Review (IJAS), 033-039.

Payton,,C.. Bartlett, R., 2008: Biomechanical evaluation of movement in sport and exercise: the British Association of Sport and Exercise Science guide. Taylor \& Francis e-Library.

Precerutti, M., Garioni, E., Madonia, L., Draghi, F., 2010: US anatomy of the shoulder: Pictorial essay. Journal of Ultrasound $\mathrm{xx}, 1-9$.

Sims, N.A., Gooi, J.H., 2008: Bone remodeling: Multiple cellular interactions required for coupling of bone formation and resorption. Semin. Cell. Dev. Biol. 19(5), 444-451.

Tempelaere, C., Pierrart, J., Lefèvre-Colau, M., Vuillemin, V., Cuénod, C., Hansen, U., Mir, O., Skalli, W., Gregory, T., 2016: Dynamic Three-Dimensional Shoulder Mri during Active Motion for Investigation of Rotator Cuff Diseases. PLoS ONE 11(7), e0158563.

Yun, G., Kang, Y., Ahn, J.M., Lee, E., Lee, J.W., Oh, J.H., Kang, H.S., 2018: A Comprehensive Review of Shoulder CT Morphometry: What Surgeons Wants to Know. J. Korean Soc. Radiol. 78(4), 265-278.

Zdero, R., 2017: Experimental Methods in Orthopaedic Biomechanics. Elsevier.

Zhibin, S., Tianyu, Ch.N., Yijun, N., 2018: A New Skeleton Model and the Motion Rhythm Analysis for Human Shoulder Complex Oriented to Rehabilitation Robotics. Applied Bionics and Biomechanics, 2719631. Hindawi.

Zotin, A., Simonov, K., Kabaev, E., Kurako, M., Matsulev, A., 2020: Evaluation of Shoulder Joint Data Obtained from CONTREX Medical System. Intelligent Decision Technologies. Proceedings of the 12th KES International Conference on Intelligent Decision Technologies (KES-IDT 2020), 155-165. Springer. 